\title{
Mimicking the niche: cytokines expand muscle stem cells
}

\author{
Cell Research (2015) 25:761-762. doi:10.1038/cr.2015.78; published online 26 June 2015
}

Muscle stem cells (MuSCs) have long been considered to be potential therapeutic vehicles for diseases of muscle such as muscular dystrophies. A recent study published in Cell Research by $\mathrm{Fu}$ et al. reveals that recapitulating in vitro the in vivo microenvironment of MuSCs that occurs during muscle regeneration might be a major step towards translation.

Muscle stem cells (MuSCs) reside in a quiescent state in vivo within a microenvironment, or niche, where they are localized underneath the basal laminae of muscle fibers [1]. Following muscle injury, MuSCs are activated, proliferate, and ultimately differentiate to repair the tissue. Moreover, during regeneration, MuSCs restore their original pool, with a subset returning to quiescence and occupying the niche by self-renewal [1]. When MuSCs are freshly isolated as quiescent stem cells from a given muscle and immediately transplanted into a host, they show a remarkable capacity to expand, engraft and eventually repair the tissue [2]. By contrast, MuSCs that have been expanded in vitro show a markedly diminished efficacy of transplantation $[2,3]$. This is particularly relevant because isolating MuSCs from a donor results in a limited number of cells, and their expansion in vitro is almost certainly necessary for most therapeutic applications. Therefore, improvement in methodologies that would allow for the expansion of MuSCs in vitro without a substantial loss of capacity to engraft and contribute to muscle repair and regeneration following transplantation would be an important advance in the field.

A growing body of evidence indicates that strategies that mimic the in vivo microenvironment may be an effective approach to the development of culture systems that are capable of sustaining stem cell growth with limited functional loss $[4,5]$. Artificial niches based on materials that mimic the mechanical and biochemical properties of the niche have been shown to preserve MuSC functionality in vitro as assessed by enhanced potency of engraftment following transplantation [6]. In addition, supplementing culture media with factors that enhance stem cell function, while not necessarily recapitulating the in vivo niche, may likewise enhance the growth of stem cells in vitro while retaining potency [7]. For example, from a screen of 2400 small molecules, Forskolin was identified as a compound that was able to promote the proliferation of MuSCs and their ability to engraft in vivo [8]. Likewise, an inhibitor of the $\mathrm{p} 38 \alpha / \beta$ MAPK was reported to ameliorate self-renewal defects of MuSCs from aged mice, resulting in enhanced potency after transplantation $[9,10]$.

With the goal of optimizing culture conditions, by mimicking the endogenous microenvironment, to promote the expansion of MuSCs without loss of potency, Fu and coworkers from Ping Hu's laboratory recently reported an important advance. In a study published in Cell Research, these authors identified a cocktail of four cytokines, IL-1 $\alpha$, IL13 , TNF- $\alpha$, and IFN- $\gamma$ that is sufficient to promote MuSC proliferation and maintain their undifferentiated status even after 20 passages in vitro [11]. The authors first characterized the dynamic changes in the MuSC microenvironment after injury that are associated with effective muscle regeneration. Flow cytometry analysis of cells present in the regenerating tissue revealed large numbers of $\mathrm{CD}^{+} \mathrm{T}$ cells 3-5 days after the injury. These observations are consistent with previous findings suggesting a role of inflammatory cells, M2 macrophages and eosinophils in particular, in facilitating muscle regeneration [12]. Fu et al. confirmed a major role that $\mathrm{T}$ cells play in regeneration by showing that transplantation of $\mathrm{T}$ cells rescued the muscle regeneration defect not only in FoxN1 $1^{--}$mice that lack T cells but also in $\mathrm{Ragl}^{-/}$mice that lack both $\mathrm{T}$ and $\mathrm{B}$ cells.

After isolation, MuSCs in culture rapidly lose their potential for growth without loss of functional capacity and show instead a propensity to differentiate during in vitro expansion $[2,3]$. By adding $T$ cells or $T$ cell conditioned medium to MuSC cultures, Fu et al. found that MuSCs were able to undergo extensive expansion and maintain exponential growth kinetics through 20 passages. Furthermore, MuSCs expanded in T cell conditioned medium and transplanted into irradiated muscles were able to engraft efficiently and home properly to the MuSC niche. In keeping with this latter observation, these culture conditions were also able to allow a vast majority of the MuSCs to maintain phenotypic characteristics of freshly isolated MuSCs during in vitro expansion. Specifically, through serial passages, $>80 \%$ of the cells continued to be characterized as $\mathrm{Pax} 7^{\text {high }} / \mathrm{MyoD}^{\text {low }}$ by immunophenotyping, with Pax 7 being a marker of quiescent and early activated MuSCs and MyoD being a marker of more differentiated MuSCs and their progeny. In conventional culture conditions, MuSCs rapidly become Pax $7^{\text {low }}$ MyoD ${ }^{\text {high }}$. Interestingly, gene expres- 
sion profiles showed that expression of several genes predominantly expressed in quiescent MuSCs was unchanged in MuSCs expanded in vitro in T cell conditioned medium, pointing out that these MuSCs retained at least some of the molecular features observed in freshly isolated MuSCs.

The role of cytokines in promoting muscle regeneration has been extensively investigated, and many inflammationrelated factors have been reported to positively influence muscle regenerative capacity [12]. Based on the hypothesis that one of the effects of factors secreted by $\mathrm{T}$ cells is to promote the proliferation of MuSCs during regeneration, and based on the premise that such factors may help to promote proliferative expansion of undifferentiated MuSCs in vitro, Fu et al. set out to identify such factors. Starting from a large set of cytokines selected based on the criteria of high transcript levels in T cells and high protein levels in $\mathrm{T}$ cell conditioned medium, Fu et al. narrowed the list to a combination of four cytokines (IL$1 \alpha$, IL-13, TNF- $\alpha$, and IFN- $\gamma$ ) that was sufficient to promote the proliferative expansion of undifferentiated MuSCs.
This cocktail, when injected into regenerating muscle of $\mathrm{Rag} \mathrm{I}^{-/-}$mice, was able to rescue both the diminished MuSC proliferation and the impaired regenerative response, phenocopying the benefits seen with $T$ cell transplantation. Importantly, the cytokine cocktail, when added to MuSC medium in vitro, promoted the proliferative expansion of MuSCs to a greater degree than the addition of $\mathrm{T}$ cell conditioned medium, maintaining Pax 7 expression in each passage. MuSCs expanded in the presence of these four cytokines and then transplanted into muscles of recipient mice were able to efficiently engraft (Figure 1), repopulate their niche, and adopt the features of endogenous MuSCs as assessed by their ability to undergo activation and self-renewal in secondary injury experiments. The authors also found that MuSCs expanded with the cytokine cocktail were able to participate in muscle regeneration at levels comparable to freshly isolated MuSCs, providing further support for the conclusion that MuSCs expanded in vitro in the presence of the cytokines retained characteristics of undifferentiated progenitors.

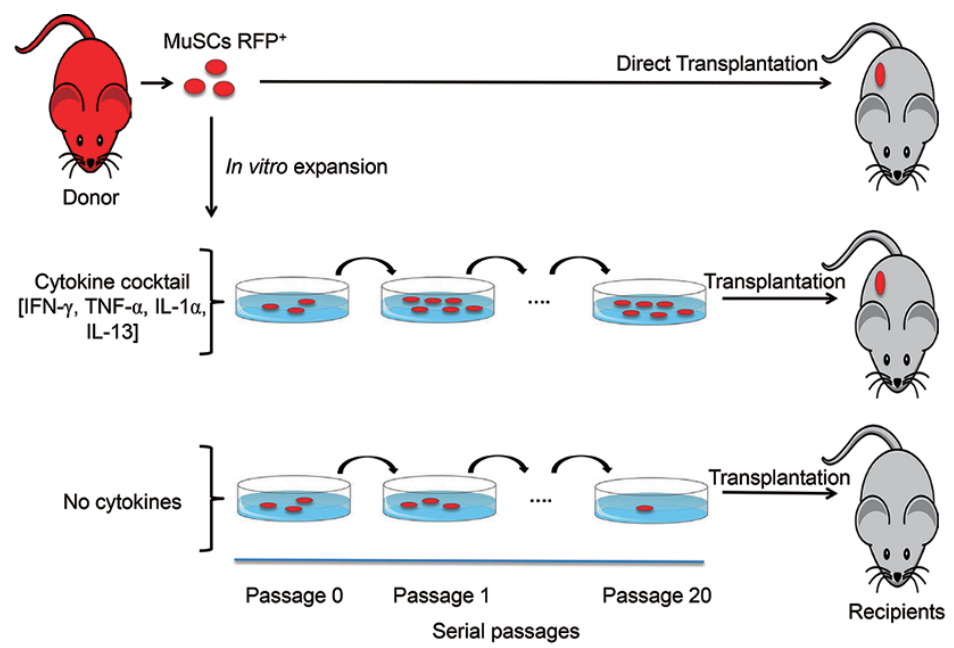

Figure 1 Cytokine cocktail expands MuSCs in vitro while preserving transplantation potency. Schematic that shows the comparison in transplantation potency among three cell populations: freshly isolated MuSCs; MuSCs cultured without the cytokine cocktail; and MuSCs cultured with the cocktail. MuSCs were isolated from mice expressing the reporter red fluorescence protein (RFP). After transplantation into recipient muscles, the fates of MuSCs were detected histologically as muscle fibers and satellite cells that stained positively for RFP.
In summary, $\mathrm{Fu}$ and co-authors present a very promising method to efficiently expand MuSCs without apparent loss of function. To address the translational value of their methods in human MuSCs, it will be important to test the ability of this (or a related) cytokine cocktail to promote the proliferative expansion without differentiation of human MuSCs. It would still be necessary to solve problems such as gene correction (for autologous cells), immunological barriers (for related or especially unrelated donor cells), and cell delivery challenges to use in vitro expanded human MuSCs as therapeutic vehicles for muscular dystrophies. Nevertheless, the findings by $\mathrm{Fu}$ et al. represent an important step in that direction.

\section{Marco Quarta ${ }^{1,2,3}$, Thomas A Rando ${ }^{1,2,3}$}

${ }^{1}$ Department of Neurology and Neurological Sciences, Stanford University School of Medicine, Stanford, CA 94305, USA; ${ }^{2}$ Paul F. Glenn Center for the Biology of Aging, Stanford University School of Medicine, Stanford, CA 94305, USA; ${ }^{3}$ Center for Tissue Regeneration, Repair, and Restoration, Veterans Affairs Palo Alto Health Care System, Palo Alto, CA 94304, USA Correspondence: Thomas A Rando E-mail: rando@stanford.edu

\section{References}

1 Collins CA, Olsen I, Zammit PS, et al. Cell 2005; 122:289-301

2 Montarras D, Morgan J, Collins C, et al. Science 2005; 309:2064-2067.

3 Sacco A, Doyonnas R, Kraft P, et al. Nature 2008; 456:502-506.

4 Scadden DT. Cell 2014; 157:41-50.

5 Lane SW, Williams DA, Watt FM. Nat Biotechnol 2014; 32:795-803.

6 Gilbert PM, Havenstrite KL, Magnusson KEG, et al. Science 2010; 329:1078-1081.

7 Parker MH, Tapscott SJ. Curr Protoc Stem Cell Biol 2013; Chapter 2:Unit 2C.4.

$8 \mathrm{Xu}$ C, Tabebordbar M, Iovino S, et al. Cell 2013; 155:909-921.

9 Bernet JD, Doles JD, Hall JK, et al. Nat Med 2014; 20:265-271

10 Cosgrove BD, Gilbert PM, Porpiglia E, et al. Nat Med 2014; 20:255-264.

11 Fu X, Xiao J, Wei Y, et al. Cell Res 2015; 25:655-673.

12 Aurora AB, Olson EN. Cell Stem Cell 2014; 15:14-25. 